\title{
EFEITOS DA TERAPIA DO ESPELHO NA FUNCIONALIDADE EM PACIENTES COM ACIDENTE VASCULAR CEREBRAL: REVISÃO INTEGRATIVA
}

\author{
EFFECTS OF MIRROR THERAPY ON FUNCTIONALITY IN PACIENTS WITH STROKE: \\ INTEGRATIVE REVIEW
}

DOI: $10.16891 / 2317-434 X . v 10 . e 1 . a 2022 . p p 1255-1259$

Recebido em: 10.11 .2021 | Aceito em: 10.11.2021

Camila Alves Caetano e Daiane Pontes Leal Lira

Centro Universitário Doutor Leão Sampaio

\begin{abstract}
RESUMO
O acidente vascular cerebral (AVC) conceitua-se como um distúrbio neurológico agudo de duração maior que 24 horas, apresentando sintomatologias relacionadas às regiões focais e globais do cérebro. Dentre os métodos de tratamento disponíveis, destaca-se a terapia do espelho, utilizada com objetivo principal de melhora da mobilidade por meio da retroalimentação visual, auxiliando na construção da plasticidade cerebral e consequente reaprendizado motor. Este estudo objetiva verificar os efeitos da Terapia do Espelho em pacientes com sequelas de acidente vascular cerebral. Essa pesquisa apresenta-se como uma revisão integrativa, com abordagem descritiva. Na coleta de dados realizada entre setembro e outubro de 2021, utilizou-se das plataformas digitais PUBMED, Biblioteca Virtual em Saúde (BVS) e o banco de dados Physiotherapy Evidence Database (PEDro). Foram selecionados 5 artigos que correspondiam com os critérios de inclusão, exclusão e elegibilidade. A partir da análise dos artigos selecionados, relata-se que a terapia do espelho proporciona resultados significativos na melhora de mobilidade em membros superiores, aumentando sua funcionalidade e proporcionando maior qualidade de vida e, em membros inferiores o método proporciona melhora na espasticidade, funcionalidade, aumento de força e velocidade ao caminhar, porém deve-se ressaltar que a terapia não se apresentou como melhor técnica na maioria dos estudos. Observou-se neste estudo que a terapia do espelho possui bons resultados com relação ao aumento de funcionalidade em pacientes com sequelas de AVC, apesar de não ser, de acordo com o estudo, a melhor técnica para esse objetivo.
\end{abstract}

Palavras-chave: Acidente Vascular Cerebral; Fisioterapia; Reabilitação Neurológica.

\begin{abstract}
The stroke is a acute neurological disorder which lasts more than 24 hours, presenting symptoms related to focal and global regions of the brain. Between the available methods of treatment, the mirror therapy is used with the purpose of incresing mobility through visual feedback, helping the construction of neuroplasticity and motor relearning. This study analyse the mirror therapy effects in pacients with stroke. This study presents itself as a integrative review, with descriptive approach. In the data collection carried out between September and October of 2021, the digital platforms PUBMED, BVS and the database Physiotherapy Evidence Database (PEDro) were used for the research. It was selected 5 articles that matched the inclusion, exclusion and eligibility criteria. From the analysis of selected articles, it is reported that mirror therapy provides significant results in improving mobility in the upper limbs, increasing their functionality and providing a better quality of life and, in the lower limbs, the method provides improvement in spasticity, functionality, increase in strength and speed when walking, but it should be noted that therapy was not presented as the best technique in most studies. It was observed in this study that mirror therapy has good results in terms of increased functionality in patients with stroke, although it is not, according to the study, the best technique for this purpose.
\end{abstract}

Keyword: Stroke; Physiotherapy; Neurological Rehabilitation. 
V.10 N.1 (2022) ISSN: 2317-434X

\section{INTRODUÇÃO}

O acidente vascular cerebral (AVC) pode ser conceituado como um distúrbio neurológico agudo com duração maior que 24 horas e que apresenta sintomatologias relacionadas às regiões focais e globais do cérebro. (BRASIL, 2016; ASSIS, 2012). No Brasil, o AVC representa a segunda maior causa de morte, e terceiro lugar como maior fator incapacitante entre os brasileiros, tornando-se um caso grave de saúde pública, gerando um grande impacto socioeconômico ao país (BRASIL, 2019).

Dentre os métodos de tratamento disponíveis, destaca-se a terapia do espelho, técnica utilizada com objetivo principal de melhora da mobilidade do paciente e que utiliza como instrumento uma caixa com um espelho onde o paciente se coloca de forma à o membro saudável estar de um lado do espelho e o membro lesionado do outro, sendo instruído a realizar movimentos com os dois membros e, mesmo que o membro afetado não esteja realizando o movimento corretamente, o reflexo do membro sadio trará a ilusão de que ambos os membros se movem de forma correta, o que ajuda na construção da plasticidade cerebral através da retroalimentação visual, auxiliando assim, na reabilitação do paciente (RAMACHANDRAN; ALTSCHULER, 2009).

Essa ilusão gerada pelo espelho ativa tanto os sistemas visual e motor, como também o cinemático e o proprioceptivo, formando uma conexão entre as áreas prémotora e visual por meio do aumento do input sensorial, auxiliando no reaprendizado motor através das etapas de observação da ação, imagem motora e imitação que a técnica apresenta a partir da observação do reflexo no espelho, a imagem do membro sadio realizando o movimento corretamente e ao tentar realizar o movimento com o membro afetado, respectivamente (ASSIS, 2012).

Sendo assim, o presente artigo possui como objetivo geral verificar os efeitos da Terapia do Espelho em pacientes com sequelas de acidente vascular cerebral através de uma revisão integrativa.

\section{MÉTODO}

$\mathrm{O}$ presente estudo trata-se de uma revisão integrativa, com abordagem descritiva. Realizou-se a coleta de dados nas bibliotecas virtuais PUBMED e BVS e no banco de dados PEDro, durante o período de setembro à outubro de 2021. Como critérios de elegibilidade, foram selecionados somente artigos completos, publicados em revistas científicas, que apresentem como metodologia os ensaios clínicos realizados nos últimos cinco anos e no idioma português ou inglês. Já como critérios de inclusão, pode-se citar artigos que apresentem pacientes adultos e/ou idosos que tiveram o acidente vascular cerebral, protocolo de fisioterapia com a utilização da terapia do espelho e resultados concluídos e, como critérios de exclusão, ressalta-se artigos que apresentem pacientes com acidente vascular transitório e que não contenha resultados finalizados.

Na BVS, utilizou-se o cruzamento dos descritores "fisioterapia", "acidente vascular cerebral" e "reabilitação neurológica", usando-se dos operadores booleanos AND e OR. Já na PUBMED, os descritores "stroke", "neurological rehabilitation" e "stroke rehabilitation" foram utilizados em conjunto com o operador booleano AND e OR, respectivamente e, por último, na PEDro realizou-se a pesquisa com os termos mirror therapy e stroke.

\section{RESULTADOS E DISCUSSÃO}

Foram encontrados, ao todo, 380 artigos nas bases de dados e, após todo o processo de seleção citado anteriormente, incluiu-se 5 artigos para o presente estudo apresentados na tabela a seguir.

Tabela 1. Apresentação das informações mais relevantes sobre os artigos selecionados para o estudo.

\begin{tabular}{llllll}
\hline ARTIGO & AUTOR/ANO & METODO & AMOSTRA & INTERVENÇÃO & RESULTADOS \\
\hline Unilateral & Daniel Simpson; & Estudo piloto & 31 participantes & A intervenção do grupo O grupo intervenção \\
dorsiflexor & Monika & paralelo & com sequelas de & somente de treinamento apresentou aumento \\
strengthening & Ehrensberger; & controlado & AVC crônico de força (grupo de força no membro & de \\
with mirror & Frances Horgan; & randomizado & foram & controle) consistiu em não treinado, ao \\
therapy to & Catherine Blake; & com avaliador & escolhidos para um programa de contrário do grupo \\
improve & David Roberts; & cego para a & o estudo: grupo treinamento de força controle que não \\
motor & Patrick & alocação do & controle (15) e unilateral isométrico apresentou aumento \\
function after & Broderick; & tratamento. & grupo & aplicado somente para o de força em ambos \\
stroke: A & Kenneth & & experimental & membro menos afetado. os membros. Os dois \\
\hline
\end{tabular}


pilot

randomized

study
Monaghan; 2019.

(n)

\section{(16).}

(16)

Esse protocolo foi seguido 3 vezes por semana por 4 semanas. Já o grupo experimental realizou o mesmo protocolo, porém com o acréscimo do espelho colocado no plano sagital do participante.

KNK Fong; KH $1^{\circ}$ parte: ensaio Ting; CCH Chan; randomizado LSW Li; 2019.

controlado simples-cego $2^{\circ}$ parte: medição transversal de eletroencefalo grafia (EEG)

$1^{\circ}$ parte: 101 participantes: 51 no grupo da terapia do espelho e 50 no treinamento bilateral MMSS. $2^{\circ}$ parte: 20 participantes: terapia do
espelho e 9 do grupo controle.

$1^{\circ}$ parte: A intervenção para ambos os grupos consistiu em um programa de exercícios bilaterais que incluiu 5 movimentos de MMSS.

A única diferença entre os grupos foi o uso do espelho no plano sagital dos participantes no grupo da terapia do espelho.

$2^{\circ}$ parte: realização da EEG com o paciente

\section{Mirror}

therapy with bilateral arm training for hemiplegic

1257pper extremity motor functions in patients with chronic stroke realizando movimentos do protocolo de tratamento.

Um ensaio cego único randomizado controlado.

Using

Gesture

Recognition

for Upper

Limb

Function,

Neck

Discomfort,

and Quality

of Life After

Chronic

Stroke:

A

SingleBlind

Randomized

Controlled

Trial

\begin{tabular}{llrl} 
A randomized & \multicolumn{2}{l}{ Shafqatullah Jan; } & Ensaio \\
control trial & Aatik & Arsh; & controlado \\
comparing & Haider & Darain; & randomizado. \\
the effects of & Shehla & Gul; & \\
motor & 2019. & & \\
relearning & & & \\
programme & & &
\end{tabular}

Um total de 36 O grupo de terapia do participantes com AVC terapia com dispositivo crônico foram baseado na terapia do divididos de espelho com utilização forma de movimentação 3D, randomizada em já o grupo da terapia do 3 grupos: GR espelho convencional terapia do realizou a terapia do espelho (12); espelho de forma geral espelho submetido à terapia convencional simulada.

(12); grupo Cada grupo foi controle (12). submetido à 15 sessões de 30 minutos de duração, 3 dias da semana por 5 semanas.

Participaram do O grupo de intervenção estudo 66 realizou programa de indivíduos que reaprendizado motor foram separados enquanto o grupo em dois grupos: controle realizou a Grupo de terapia do espelho. O intervenção (33) grupo de intervenção terapia do e o grupo controle foi grupos apresentaram redução significante na espasticidade do membro não treinado (mais afetado) e houve aumento médio da velocidade ao andar no grupo da terapia do espelho em comparação ao grupo controle.

A terapia do espelho se mostrou mais válida em melhorar a função de região distal de MMSS. Além disso, o feedback visual pelo espelho pode proporcionar a ativação do córtex sensório-motor contralateral, gerando uma simetria maior do cérebro durante a recuperação da hemiplegia pós avc.

A função de extremidade de MMSS e qualidade de vida tiveram melhora significativa no grupo terapia do espelho GR em comparação com o grupo controle. As mudanças de desconforto de cervical no grupo de terapia do espelho convencional e grupo controle foram significativamente melhores do que no grupo terapia do espelho GR.

Tanto o Programa de Reaprendizado Motor como a Terapia do Espelho apresentaram resultados positivos em relação à melhora 
V.10 N.1 (2022) ISSN: 2317-434X

and mirror
therapy for
improving
1258pper
limb motor
functions in
stroke
patients

\begin{tabular}{|c|c|c|}
\hline $\begin{array}{l}\text { Unilateral } \\
\text { Strength }\end{array}$ & $\begin{array}{l}\text { Monika } \\
\text { Ehrensberger; }\end{array}$ & $\begin{array}{l}\text { Estudo piloto } \\
\text { randomizado }\end{array}$ \\
\hline Training and & Daniel Simpson; & controlado de \\
\hline Mirror & Patrick & grupo paralelo. \\
\hline Therapy & Broderick; & \\
\hline Patients With & Catherine Blake; & \\
\hline Chronic & Frances Horgan; & \\
\hline Stroke A Pilot & Paula Hickey; & \\
\hline Randomized & Joanne O'Reilly; & \\
\hline Trial & and Kenneth & \\
\hline & Monaghan; 2019. & \\
\hline
\end{tabular}

\author{
e grupo controle \\ (33).
}

32 participantes com sequelas de AVC crônico foram submetidos à pesquisa: grupo da intervenção (17) e grupo controle (15) realizou exercícios de tarefas específicas como exercícios de alcance e manipulação de objetos, já o grupo controle realizou a terapia do espelho convencional.

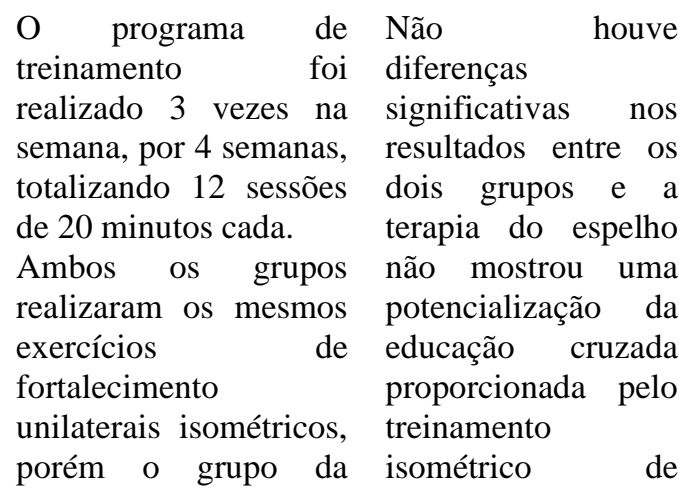

terapia do espelho membro superior. utilizou-se do espelho no plano sagital dos participantes enquanto o grupo controle realizou os exercícios sem o uso do espelho. motora e funcional de membro superior, porém o grupo da intervenção obteve resultados melhores em comparação ao grupo controle.

Fonte: Dados da pesquisa (2021).

Os estudos de Simpson et al (2019) e Ehrensberger et al (2019) apresentam muitas similaridades, principalmente no protocolo de tratamento e na amostra, porém o primeiro estudo foi realizado em MMII e o segundo em MMSS. A partir disso, é importante ressaltar que ambos os estudos obtiveram resultados contraditórios, mesmo apresentando protocolos iguais, abrindo uma indagação para novos estudos que possibilitem maiores explicações sobre o ocorrido.

Destaca-se também o estudo de Choi (2019) que apresentou a conexão entre a terapia do espelho e a utilização de jogos em 3D realizados de forma conjunta, potencializando os efeitos da terapia do espelho na mobilidade de pacientes com sequelas de AVC como o próprio artigo demonstra.

Em uma revisão sistemática realizada por Thieme et al (2018), obteve-se evidência moderada com relação à melhora de função motora a das atividades de vida diária de pacientes pós AVC submetidos à terapia do espelho, indo de encontro com o presente estudo que mostrou resultados positivos com relação ao aumento de mobilidade e funcionalidade dos pacientes pós AVC, porém a técnica não se apresentou como melhor forma de tratamento.

\section{CONSIDERAÇÕES FINAIS}

A terapia do espelho possui bons resultados com relação ao aumento de funcionalidade em pacientes com sequelas de AVC, apesar de não ser, de acordo com o estudo, a melhor técnica para esse objetivo, ainda assim é válido a sua utilização pelos seus benefícios de ser um método de baixo custo, fácil acesso e possibilidade de realização do paciente em domicílio. 


\section{REFERÊNCIAS}

ASSIS, R. D. (ed.). Condutas práticas em fisioterapia neurológica. 1 ed. Barueri, SP: Manole, 2012.

Brasil. Ministério da Saúde. Secretaria de Atenção à Saúde. Departamento de Ações Programáticas Estratégicas. Diretrizes de atenção à reabilitação da pessoa com acidente vascular cerebral. Brasília: Ministério da Saúde, 2016. 72p.: il.

CHOI, Ho-Suk; SHIN, Won-Seob; BANG, Dae-Hyouk. Mirror therapy using gesture recognition for upper limb function, neck discomfort, and quality of life after chronic stroke: A single-blind randomized controlled trial. Medical science monitor: international medical journal of experimental and clinical research, v. 25, p. 3271-3278, maio de 2019.

EHRENSBERGER, Monika et al. Unilateral strength training and mirror therapy in patients with chronic stroke: A pilot randomized trial. American journal of physical medicine \& rehabilitation, v. 98 , n. 8 , p. 657-665, agosto de 2019.

FONG, K.N.K; TING, K.H; CHAN, C.C.H; LI, L.S.W. Mirror therapy with bilateral arm training for hemiplegic upper extremity motor functions in patients with chronic stroke. Hong Kong Medical Journal, v. 25, n.1, fevereiro de 2019.
JAN, Shafqatullah; ARSH, Aatik; DARAIN, Haider; GUL, Shehla. A randomized control trial comparing the effects of motor relearning programme and mirror therapy for improving upper limb motor functions in stroke patients. JPMA, v. 69 , n. 9, p. 1242-1245, setembro de 2019.

RAMACHANDRAN, V. S; ALTSCHULER, E. L. The use of visual feedback, in particular mirror visual feedback, in restoring brain function, Brain, v. 132, n. 7, p. 1693-1710, julho de 2009.

SIMPSON, Daniel et al. Unilateral dorsiflexor strengthening with mirror therapy to improve motor function after stroke: A pilot randomized study. Physiotherapy research international, v. 24, n. 4, p. e1792, 2019.

THIEME, H. et al. Mirror therapy for improving motor function after stroke (Review). Cochrane Database of Systematic Reviews, v. 7. Art. No.: CD008449. DOI: 10.1002/14651858.CD008449.pub3, 2018.

https://www.gov.br/saude/pt-br <Disponível em 24/12/2019, https://www.gov.br/saude/ptbr/assuntos/noticias/ministerio-da-saude-cria-linha-decuidados-para-tratar-avc> Acessado em março de 2021. 